\title{
Application of LCD-SVD Technique and CRO-SVM Method to Fault Diagnosis for Roller Bearing
}

\author{
Songrong Luo, ${ }^{1,2}$ Junsheng Cheng, ${ }^{2}$ and HungLinh $\mathbf{A o}^{3,4,5}$ \\ ${ }^{1}$ College of Mechanical Engineering, Hunan University of Arts and Science, Changde 415003, China \\ ${ }^{2}$ College of Mechanical and Vehicle Engineering, Hunan University, Changsha 410082, China \\ ${ }^{3}$ Institute for Computational Science, Ton Duc Thang University, Ho Chi Minh 70000, Vietnam \\ ${ }^{4}$ Faculty of Civil Engineering, Ton Duc Thang University, Ho Chi Minh 70000, Vietnam \\ ${ }^{5}$ Faculty of Mechanical Engineering, Industrial University of Ho Chi Minh City, Ho Chi Minh 70000, Vietnam
}

Correspondence should be addressed to Songrong Luo; luosr7351@sina.com

Received 5 December 2014; Accepted 19 March 2015

Academic Editor: Anindya Ghoshal

Copyright (c) 2015 Songrong Luo et al. This is an open access article distributed under the Creative Commons Attribution License, which permits unrestricted use, distribution, and reproduction in any medium, provided the original work is properly cited.

Targeting the nonlinear and nonstationary characteristics of vibration signal from fault roller bearing and scarcity of fault samples, a novel method is presented and applied to roller bearing fault diagnosis in this paper. Firstly, the nonlinear and nonstationary vibration signal produced by local faults of roller bearing is decomposed into intrinsic scale components (ISCs) by using local characteristic-scale decomposition (LCD) method and initial feature vector matrices are obtained. Secondly, fault feature values are extracted by singular value decomposition (SVD) techniques to obtain singular values, while avoiding the selection of reconstruction parameters. Thirdly, a support vector machine (SVM) classifier based on Chemical Reaction Optimization (CRO) algorithm, called CRO-SVM method, is designed for classification of fault location. Lastly, the proposed method is validated by two experimental datasets. Experimental results show that the proposed method based LCD-SVD technique and CRO-SVM method have higher classification accuracy and shorter cost time than the comparative methods.

\section{Introduction}

A roller bearing is widely used in industrial applications. Failures of roller bearing generally lead to malfunction of system and even terrible accidents. According to statistics, 30-40\% of failure is produced by the bearing of rotating machinery [1]. Therefore, research on roller bearing fault diagnosis has important significance in industrial application.

Fault diagnosis of rotating machinery mainly includes two key steps. One key step is feature extraction. Using suitable vibration signal processing approach, fault feature could be generally extracted $[2,3]$. Recently, singular value decomposition (SVD) technique has been applied to fault diagnosis of rotating machinery [4-6]. However, SVD technique would suffer complex selection of reconstruction parameters [6]. Empirical mode decomposition (EMD) method [7] is excellent time-frequency analysis approach to deal with nonstationary vibration signals produced by machinery fault diagnosis, but EMD method still has some drawbacks, such as end effect and mode mixing [8-11]. We have developed a new time-frequency analysis approach, namely, local characteristic-scale decomposition (LCD) method [12, 13]. Similar to EMD method, LCD method can decompose a complex signal into several intrinsic scale components (ISCs). It is most important point that LCD is superior to EMD in running time, restraining the end effect and relieving mode mixing $[12,13]$. Therefore, LCD method is used as vibration signal processing approach in this paper. Since each ISC contains special time-scale information of original signal, the ISCs of vibration signal, which reflect nature characteristics of original signal, can be employed to construct initial fault feature matrix for SVD technique avoiding difficulty of choosing reconstruction parameters. Hence, SVD technique combining with LCD method, namely, LCDSVD, is introduced to extract the fault feature of roller bearing in this paper. 
Another key step in fault diagnosis is pattern recognition [14-18]. Support vector machine (SVM) [19] has been proven to have an excellent generalization capability and has been successfully applied to machinery fault diagnosis with insufficient fault samples. Unfortunately, SVM parameters affect classification performance $[16,17]$. Selecting appropriate parameters is always challenge for scientists. Since traditional approaches of determined parameters, such as grid algorithm [20] and cross-validation method [21], need large amount of complex calculation, some heuristic algorithms have been employed to optimize the SVM parameters, such as genetic algorithm (GA) [17], particle swarm optimization (PSO) [18], ant colony optimization (ACO) [22], and Lam and Li's developed Chemical Reaction Optimization (CRO) algorithm inspired by the nature of chemical reactions [23]. The CRO algorithm is based on the second law of thermodynamics to select feasible search space. Compared with the existing heuristic optimization algorithms, the $\mathrm{CRO}$ can reach a global optimum in a very short time [24]. Therefore, using CRO algorithm to optimize the parameters of SVM classifier, CRO-SVM method is presented to fulfil pattern recognition in roller bearing fault diagnosis.

In summary, a new fault diagnosis method based on LCD-SVD technique and CRO-SVM is proposed in this paper. The remaining part is organized as follows. Section 2 discusses the LCD method. In Section 3, LCD-SVD method is introduced. Section 4 explains the CRO method and CROSVM method. In Section 5, the fault diagnosis method for roller bearing based on LCD-SVD and the CRO-SVM is presented. In Section 6, the fault diagnosis method is applied to detect the fault location of roller bearings. The conclusions drawn from this research are given in Section 7.

\section{Local Characteristics-Scale Decomposition Method}

LCD method is a kind of perfect time-frequency analysis approach. It is suitable for nonstationary and nonlinear signal. According to this method, a complex signal can be self-adaptively decomposed into a sum of ISCs and a residue $r_{n}(t)$. That is,

$$
x(t)=\sum_{i=1}^{m} \operatorname{ISC}_{i}+r_{n}(t),
$$

where $i$ is the index of ISCs and $r_{n}(t)$ is a residue. References $[12,13]$ have described this method. Each of ISCs includes main information of different frequency bands ranging from high to low and the information will change with the original signal $x(t) . r_{n}(t)$ represents the central tendency of original signal $x(t)$.

Figure 1 shows the vibration acceleration signal of the normal bearing, inner-race fault bearing, and outer-race fault bearing, respectively. As seen from Figure 1, the vibration signals caused by roller bearing faults present nonstationary and nonlinear characteristics. As mentioned above, this kind of signal can be decomposed by LCD method. Figure 2 gives the decomposed results of the bearing vibration signal with inner-race fault. Figure 2 shows that signal is decomposed

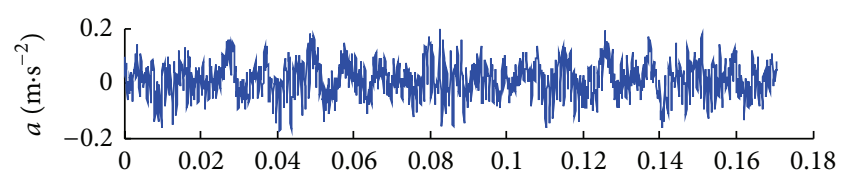

(a)

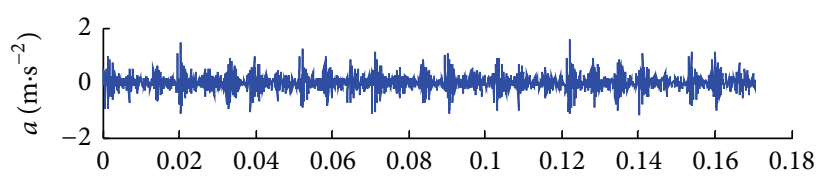

(b)

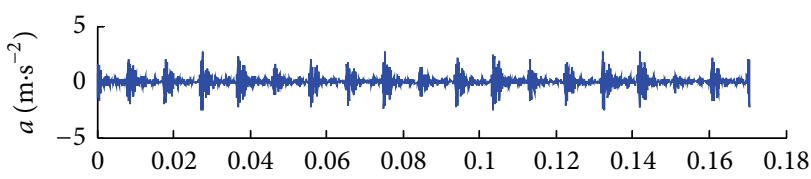

(c)

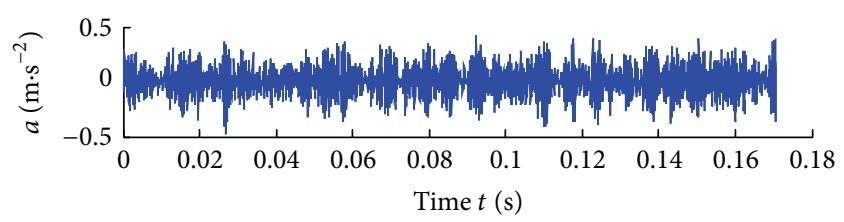

(d)

FIGURE 1: Time domain waveforms of roller bearing (a) normal, (b) inner-race fault, (c) outer-race fault, and (d) ball fault.

into some ISCs with different time scale and the characteristics of the signal can be drawn more obviously.

\section{Singular Value Decomposition Technique Based on LCD Method (LCD-SVD Technique)}

For the nonlinear time series of $N$ data points $\{x(i), i=$ $1,2, \ldots, N\}$, a trajectory matrix $\mathbf{X}_{L \times K}$ can be formed based on phase space reconstruction theory. Let window length $L$ be an integer and $K$ the number of lagged vectors $\mathbf{X}_{i}=$ $\left(x_{i}, \ldots, x_{i+K-1}\right)$, where $1<L<N, K=N-L+1,1 \leq i \leq l$. The trajectory matrix $\mathbf{X}_{L \times K}$ can be expressed as

$$
\mathbf{X}_{L \times K}=\left[\begin{array}{cccc}
x_{1} & x_{2} & \cdots & x_{K} \\
x_{2} & x_{3} & \cdots & x_{K+1} \\
\vdots & \vdots & \cdots & \vdots \\
x_{L} & x_{L+1} & \cdots & x_{N}
\end{array}\right]
$$

Based on singular value decomposition (SVD) theory, a trajectory matrix $X_{L \times K}$ can be decomposed into a series of mutually orthogonal, unit-rank, and elementary matrices by using SVD [4], that is,

$$
X_{L \times K}=\mathbf{U S V}^{T},
$$

where $\mathbf{U} \in R^{L \times L}$ and $\mathbf{V} \in R^{K \times K}$ and $\mathbf{S}$ is a $L \times K$ diagonal matrix; let $\lambda_{1}, \lambda_{2}, \lambda_{3}, \ldots, \lambda_{n}$ be nonzero diagonal elements 

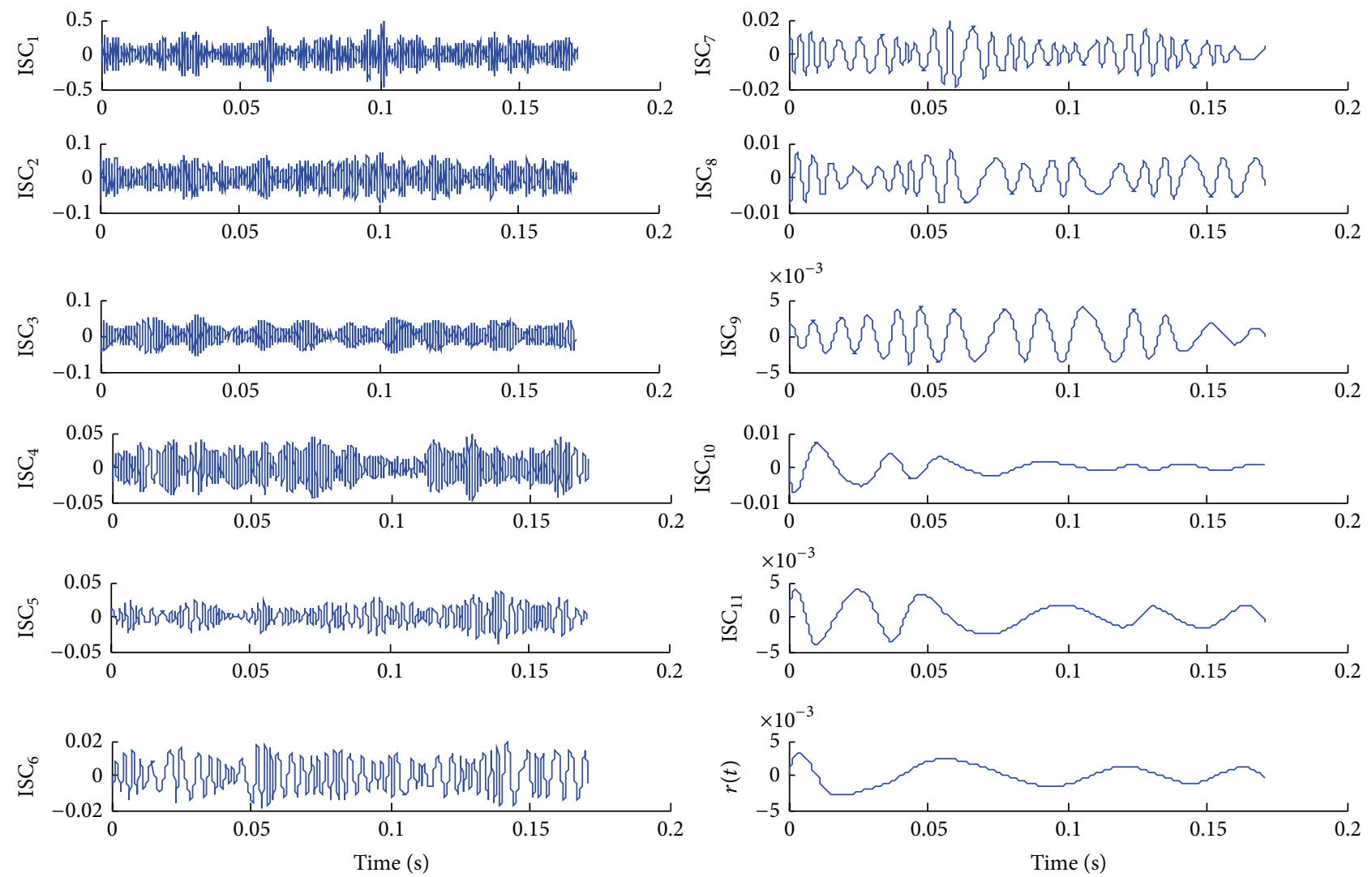

FIGURE 2: Time domain waveform of ISCs of vibration signal of roller bearing with ball fault.

arranged in decreasing order. They are called singular values of matrix $X_{L \times K}$, namely, singular spectrum, where $n=$ $\operatorname{rand}\left(X_{L \times K}\right), \lambda_{1} \geq \lambda_{2} \geq \lambda_{3} \geq \cdots \geq \lambda_{n}>0$. The number $n$ of nonzero diagonal elements reflects the complexity of frequency components in the nonlinear time series. When $n$ is bigger, the frequency components are more complex. Therefore, the singular spectrum of matrix $X_{L \times K}$ describes the characteristics of the nonlinear time series correspondingly.

However, reconstruction parameters, such as lag time and embedding dimension, would have effect on the result of SVD method. However, there is no mature theory for selecting the reconstruction parameters [5]. Targeting the nonlinear and nonstationary characteristics of vibration signals from bearing exhibiting local faults and the limitations of SVD techniques, LCD-SVD technique is presented to extract the fault feature vector in this investigation. Firstly, the nonlinear and nonstationary vibration signal is decomposed into a number of ISCs. Then, after selecting several ISCs to form a trajectory matrix, SVD is applied to obtain singular values as fault feature vector. Therefore, LCD-SVD technique should be able to extract the nonlinear and nonstationary characteristics of vibration. At the same time, this kind of method could easily avoid the difficulty of determining reconstruction parameters of SVD. Moreover, in this signal processing, because only several special ISCs are selected after analysis, the noise would be eliminated and the LCD-SVD method could improve the accuracy of feature extraction.

\section{Parameter Optimization of SVM Based on CRO}

4.1. Support Vector Machine. SVM was developed based on statistical learning theory. The basic idea of the SVM is mapping the training samples from the input space into a feature higher-dimensional space via a mapping function [19]. Suppose there is a given training sample set $G=\left\{\left(x_{i}, y_{i}\right)\right.$, $i=1,2, \ldots, l\}$, where each sample $x_{i} \in R^{d}$ belongs to a class by $y \in\{+1,-1\}$. When the training data are not linearly separable in the feature space, the target function can be expressed as follows [19]:

$$
\begin{array}{ll}
\min & \phi(\omega)=\frac{1}{2}\langle\omega \cdot \omega\rangle+C \sum_{i=1}^{l} \xi_{i} \\
\text { s.t. } & y_{i}\left(\left\langle\omega \cdot \phi\left(x_{i}\right)\right\rangle+b\right) \geq 1-\xi_{i}, \\
& \xi_{i} \geq 0, \quad i=\{1,2, \ldots, l\},
\end{array}
$$

where $\omega$ is the normal vector of the hyperplane; $C$ is a penalty parameter; $b$ is the bias that is a scalar; $\xi_{i}$ are nonnegative slack variables; $\phi(x)$ is a mapping function. By introducing a set of 
Lagrange multipliers $\alpha_{i} \geq 0$, the optimization problem can be expressed as [19]

$$
\text { Maximize } L(\omega, b, \alpha)=\sum_{i=1}^{l} \alpha_{i}-\frac{1}{2} \sum_{i, j=1}^{l} \alpha_{i} \alpha_{j} y_{i} y_{j} K\left(x_{i} \cdot x_{j}\right)
$$

Subject to $0 \leq \alpha_{i} \leq C$

$$
\sum_{i=1}^{l} \alpha_{i} y_{i}=0
$$

The decision function can be given as [19]

$$
f(x)=\operatorname{sgn}\left[\sum_{i=1}^{l} \alpha_{i} y_{i} K\left(x_{i} x\right)+b\right] \text {. }
$$

In general, the radial basis function kernel "RBF" is used as kernel functions in SVM method in machinery fault diagnosis [25]. "RBF" function is rewritten in the following equation:

$$
K\left(x, x_{i}\right)=\exp \left(-\frac{\left\|x-x_{i}\right\|^{2}}{2 \sigma^{2}}\right)
$$

where $\sigma$ is kernel parameter.

4.2. Chemical Reaction Optimization Algorithm. Chemical Reaction Optimization (CRO) algorithm is an efficient optimization technique. It mimics the interactions of molecules in chemical reaction to reach a low energy stable state in chemical reactions. The stochastic search algorithm of CRO is inspired by chemical reaction process, which transforms the unstable substances to the stable ones $[23,24]$. CRO conducts stochastic searches using a population of molecules, each of which represents a possible solution to the problem for a specific problem. A population consists of a finite number of molecules; each molecule is decided by an evaluating mechanism to obtain its potential energy. Based on this potential energy and CRO operators, a new molecule is generated. In CRO, four types of elementary reactions are included: on-wall ineffective collision, decomposition, intermolecular ineffective collision, and synthesis. The principle includes four main steps: initialization, simulation of the chemical reactions, update of the reactants, and checking of the termination criterion. The details of CRO algorithm can be seen in $[23,24]$.

4.3. CRO-SVM Method. The classification performance of support vector machine depends on its parameters. As mentioned above, Gaussian radial basis function kernel "RBF" is widely adopted as kernel function, whose parameters include a penalty factor $C$ and the standard deviation $\sigma$. Many techniques have been developed to select the SVM parameter [16-18]. In this paper, we use CRO to optimize the parameters of the SVM to improve the performance of SVM classifier.The flow chart of the CRO-SVM is shown in Figure 3.

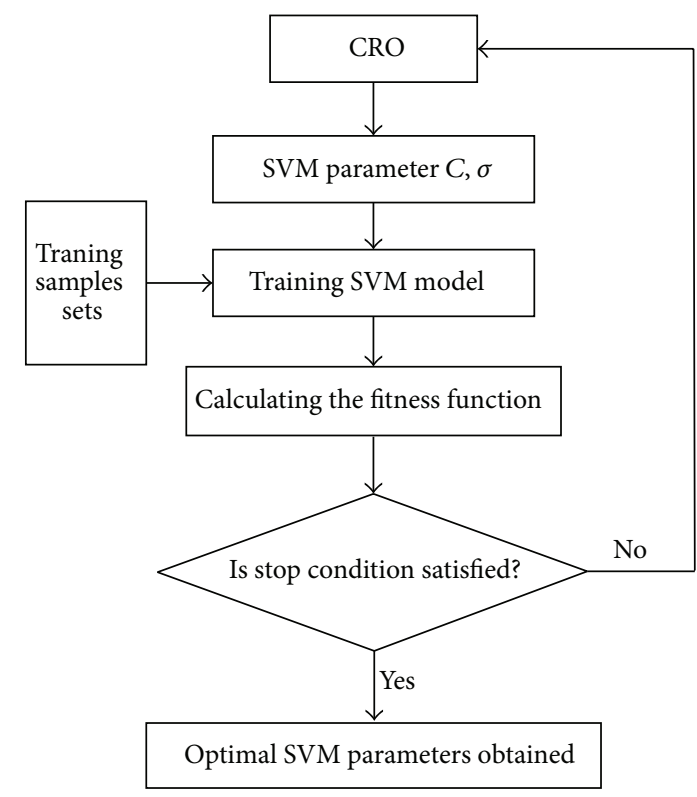

FIGURE 3: Parameter optimization flow chart of SVM based on CRO.

First of all, the objective function and fitness function should be determined according to the specific problem. Secondly, the parameters and initial value (i.e., initial reactant) should be set. Thirdly, CRO algorithm is used to optimize parameters; subsequently, training data subset is input to train CRO-SVM model. Finally, based on classification accuracy, it will be judged whether the result meets the requirements. If they meet the requirements, the optimal parameters will be output and form the optimal classifier. If they do not meet the requirements, CRO method will continue to run until classification performance of SVM classifier meets the requirements and the loop terminates.

In the proposed CRO-SVM method, these variables needing optimizing are $C$ and $\sigma$, and the fitness function is the test error of the SVM [26]. That is,

$$
f(x)=\text { Test_Error }{ }_{\mathrm{SVM}}(x),
$$

where $x=(C, \sigma)$ and the test error of SVM is defined as

$$
\text { Test_Error }_{\mathrm{SVM}}=\frac{N_{\text {error }}}{N_{\text {total test }}} \times 100 \% \text {, }
$$

where Test_Error ${ }_{\text {SVM }}$ is the test error rate. $N_{\text {error }}$ is the number of miss-classified test samples and $N_{\text {total test }}$ is the total number of test samples.

It should be noted that setting the parameters for any optimization algorithm is very important when using that algorithm. Chemical Reaction Optimization parameters influence the classification accuracy [24]. Selecting the proper ratio of reactions is a very critical issue affecting the performance of CRO. The CRO parameters were fixed with the values given in the literature [27]; that is, iterations $=50$; PopSize $=5$, MoleColl $=0.6$, InitialKE $=1,000$, KELossRate $=$ $0.01, \alpha=50, \beta=700$, and buffer $=0$. The results of each test 


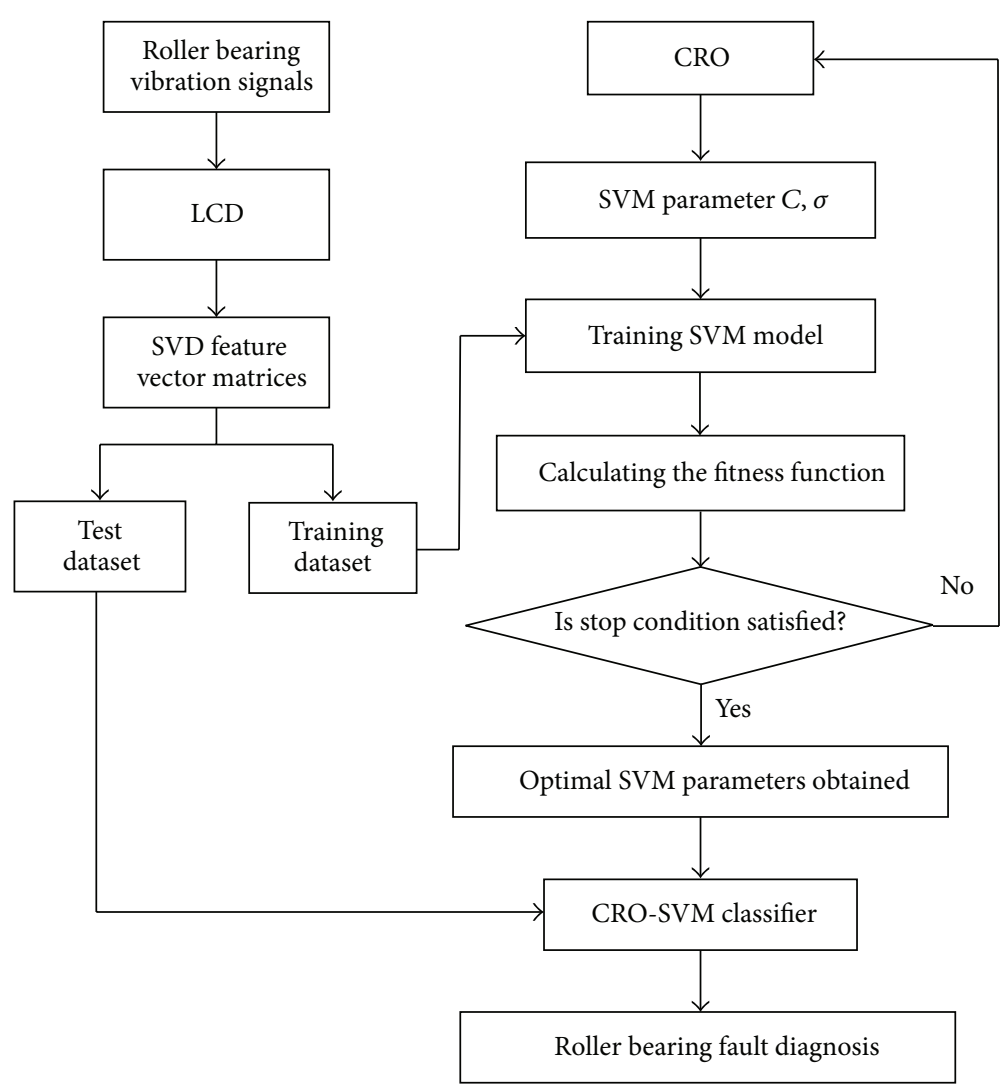

FIGURE 4: Flowchart of the roller bearing fault diagnosis method based on LCD-SVD and CRO-SVM.

are generated by averaging the error rate of the testing set in 50 trials. According to [16-18], the lower and upper bounds of the $C$ and $\sigma$ were given in $\left[2^{-14}, \ldots, 2^{14}\right]$ for the CRO-SVM, the GA-SVM, the PSO-SVM, and the CRO-SVM classifiers below.

\section{The Rotating Machinery Fault Diagnosis Method Based on LCD-SVD and CRO-SVM}

Considering feature extraction and pattern recognition methods, we proposed a new fault diagnosis method based on LCD-SVD and the CRO-SVM for roller bearing. The scheme of the proposed method is shown in Figure 4. The procedure is given as follows.

Step 1. Using vibration accelerometer, sample $N$ times at a certain sample frequency $f_{s}$ under running conditions, for example, normal condition, outer-race fault, and inner-race fault condition, respectively. And all vibration signals are taken as samples that are divided into two subsets, training samples and testing samples.

Step 2. Each sample signal is decomposed by LCD method and series of ISC can be obtained.
Step 3. Construct initial feature vector matrices $\mathbf{A}$ to each roller bearing vibration signal of each working condition according to

$$
\mathbf{A}=\left[\begin{array}{c}
\mathrm{ISC}_{1} \\
\mathrm{ISC}_{2} \\
\vdots \\
\mathrm{ISC}_{J}
\end{array}\right],
$$

where $J$ is the number of ISCs, which contain main fault information selected after detailed analysis in order to decrease computation tense and diminish the noise in the vibration signal. Hence, the characteristic of roller bearing vibration signal $x(t)$ could be extracted from the initial feature vector matrices $\mathbf{A}$.

Step 4. Obtain the singular values $\lambda$ of initial feature vector matrices $\mathbf{A}$. By applying SVD technique,

$$
\lambda=\left[\lambda_{1}, \lambda_{2}, \ldots, \lambda_{J}\right],
$$

where $\lambda_{1} \geq \lambda_{2} \geq \cdots \geq \lambda_{J}$, $J$ refer to the number of singular values.

Step 5. Design CRO-SVM classifiers. The singular values of the initial feature vector matrices $\mathbf{A}$ of the training samples 


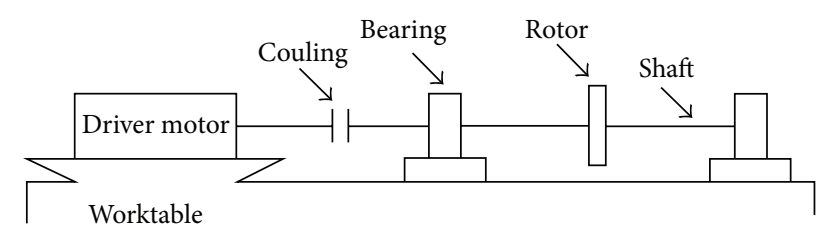

FIgURE 5: Test rig.

are used as the fault feature vectors to train the CRO-SVM classifiers.

\section{Application to Fault Diagnosis for Roller Bearing}

6.1. Data Acquisition. The first dataset has been carried out on the small test rig shown in Figure 5, which includes a motor, a coupling, a rotor, and a shaft with two roller bearings. Two types of faults are introduced in the inner race and the outer race of roller bearing (type 6311) by laser cutting slot with a width of $0.15 \mathrm{~mm}$ and a depth of $0.13 \mathrm{~mm}$. The shaft rotational frequency is $25 \mathrm{~Hz}$ and the first three resonance frequencies of the roller bearing are determined as $420 \mathrm{~Hz}, 732 \mathrm{~Hz}$, and $1016 \mathrm{~Hz}$, respectively. When local faults occur, the higher resonances would likely be excited. Hence, according to the sampling theory, the sampling frequency is set to $4096 \mathrm{~Hz}$. The vibration signals were collected by the acceleration sensor placed on the bearing seat. The roller bearings with the three conditions (normal, inner-race fault (IR fault), and outer-race fault (OR fault)) were tested, and 45 vibration signals collected in each condition were obtained, from which 30 groups were selected randomly as the training samples and the rest as testing samples.

The second dataset was downloaded from the Case Western Reserve University Bearing Data Center Website. The test stand includes a $2 \mathrm{hp}$ reliance electric motor, a torque transducer/encoder, a dynamometer, and control electronics. The sample frequency is $485063 \mathrm{~Hz}$ and motor rotating speed is $1772 \mathrm{rpm}$. In this paper, the test bearings of electrodischarge machining with fault diameters of 0.007 inches are selected. The roller bearings with the four conditions (normal, innerrace fault, outer-race fault, and ball fault) were tested, and 80 groups of vibration signals in each condition were obtained, from which 60 groups were selected at random as the training samples and the rest as the test samples.

6.2. Experiment Results. First, the vibration signals are decomposed into a number of ISCs by LCD method. It is noticed by preliminary analysis that the first four ISCs of higher frequency contain the main fault information of roller bearing. Therefore, the initial feature vector matrix $\mathbf{A}$ is established with the first four ISCs of higher frequency. Second, applying SVD to initial feature vector matrix A, the corresponding singular values can be obtained, which could be regarded as input vectors for the CRO-SVM classifier. Third, with the input vectors from training samples, the CROSVM classifiers were designed to achieve multiclassification CRO-SVM as Figure 6. Last of all, unknown samples are

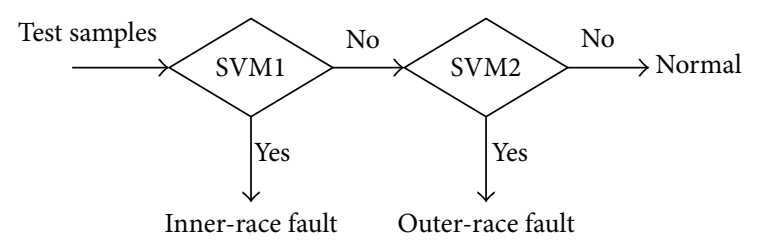

FIgURE 6: Flow chart of fault diagnosis with multiclassification SVM.

classified with CRO-SVM designed to identify roller bearing conditions and fulfill fault location. For SVM1, define the running condition with inner-race fault as $y=+1$ and the other condition as $y=-1$; thus the inner-race fault could be separated from other conditions by SVM1. Then, define the condition with outer-race fault as $y=+1$ and the other condition as $y=-1$ for SVM2; thus, the outer-race fault could be separated from other conditions by SVM2, and the rest is normal condition. In order to make a contrast, we also design GA-SVM classifier and PSO-classifier at the same time. For two datasets, respectively, the identification results with above three kinds of classifiers for the same testing samples are shown in Tables 1 and 3. Tables 2 and 4 give the part classification result of LCD-SVD-CRO-SVM classifier for two datasets, respectively.

As seen from Tables 1 and 3, when we adopt LCD-SVD technique to fulfill fault feature extraction, all three kinds of classifiers, including PSO-SVM, GA-SVM, and CRO-SVM, exhibit good classification performance. The classification success rates are above $90 \%$. This proves that LCD-SVD technique is suitable for drawing the nonlinear and nonstationary characteristics of vibration signals and effective for fault feature extraction in roller bearing fault diagnosis, due to perfect self-adaptive ability. Simultaneously, the comparative analysis results show that CRO-SVM classifier combining with LCD-SVD (noted as LCD-SVD-CRO-SVM classifier) has higher average classification success rate and much shorter cost times than the comparative methods, because the new efficient optimization algorithm, CRO algorithm, is employed to improve the performance of SVM classifier.

\section{Conclusion}

In this paper, a fault diagnosis for roller bearing method based on LCD-SVD and CRO-SVM is proposed. Firstly, LCD-SVD technique is introduced to extract the fault feature vector. Secondly, CRO-SVM method is developed for classification recognition. Lastly, two datasets of roller bearing are used to verify the proposed method. The results of both the two datasets indicate that LCD-SVD technique is suitable for nonlinear and nonstationary signal processing due to selfadaptability and effective for fault feature extraction. Furthermore, the comparative analysis shows that the CRO-SVM classifier combination with LCD-SVD method outperforms the PSO-SVM classifier and GA-SVM classifier. The proposed method can get higher accuracy rate, while cost time is much shorter than the comparative methods. In summary, this novel proposed method is efficient and feasible for 
TABLE 1: Identification results of 1st dataset obtained by LCD-SVD-CRO-SVM, LCD-SVD-GA-SVM, LCD-SVD-PSO-SVM, and LCD-SVDCRO-SVM method.

\begin{tabular}{lcccccc}
\hline Method & Training samples & Test samples & Optimal C & Optimal $\sigma$ & Cost time (s) & Identification rate $(\%)$ \\
\hline LCD-SVD-CRO-SVM1 & 45 & 15 & 3213.29 & 402.23 & 2.57 & 100 \\
LCD-SVD-GA-SVM1 & 45 & 15 & 176.08 & 38.32 & 27.10 & 100 \\
LCD-SVD-PSO-SVM1 & 45 & 15 & 168.30 & 38.31 & 12.91 & 100 \\
LCD-SVD-CRO-SVM2 & 30 & 10 & 4187.47 & 654.50 & 1.05 & 100 \\
LCD-SVD-GA-SVM2 & 30 & 10 & 107.37 & 44.98 & 12.32 & 99.3 \\
LCD-SVD-PSO-SVM2 & 30 & 10 & 4096.00 & 4.83 & 25.71 & 99.4 \\
\hline
\end{tabular}

TABLE 2: Identification results of 1st dataset based on LCD-SVD and CRO-SVM method.

\begin{tabular}{|c|c|c|c|c|c|c|c|c|}
\hline Test samples & & Faul & eature & ctor & & LCD-SVD-CRO-SVM1 & LCD-SVD-CRO-SVM2 & Identification results \\
\hline IR fault & 240.63 & 89.30 & 48.28 & 34.71 & 28.26 & $(+1)$ & & IR fault \\
\hline IR fault & 302.66 & 84.41 & 55.63 & 44.05 & 47.09 & $(+1)$ & & IR fault \\
\hline OR fault & 90.89 & 25.87 & 18.16 & 13.79 & 9.37 & $(-1)$ & $(+1)$ & OR fault \\
\hline OR fault & 72.79 & 55.61 & 45.84 & 23.41 & 19.05 & $(-1)$ & $(+1)$ & OR fault \\
\hline Normal & 55.01 & 25.37 & 18.03 & 14.16 & 13.85 & $(-1)$ & $(-1)$ & Normal \\
\hline Normal & 58.28 & 27.41 & 20.71 & 14.70 & 10.53 & $(-1)$ & $(-1)$ & Normal \\
\hline
\end{tabular}

TABLE 3: Identification results of 2nd dataset obtained by LCD-SVD-CRO-SVM, LCD-SVD-GA-SVM, LCD-SVD-PSO-SVM, and LCDSVD-CRO-SVM method.

\begin{tabular}{|c|c|c|c|c|c|c|}
\hline Method & Training samples & Test samples & Optimal C & Optimal $\sigma$ & Cost time $(\mathrm{s})$ & Identification rate (\%) \\
\hline LCD-SVD-CRO-SVM1 & 60 & 20 & 0.37805 & 0.4472 & 7.09 & 100 \\
\hline LCD-SVD-GA-SVM1 & 60 & 20 & 125.51 & 0.8445 & 16.29 & 95.2 \\
\hline LCD-SVD -PSO-SVM1 & 60 & 20 & 4082.21 & 0.8388 & 15.17 & 95.5 \\
\hline LCD-SVD-CRO-SVM2 & 45 & 15 & 3991.31 & 7.48 & 3.83 & 100 \\
\hline LCD-SVD-GA-SVM2 & 45 & 15 & 14.112 & 1.84 & 7.21 & 93.3 \\
\hline LCD-SVD -PSO-SVM2 & 45 & 15 & 902.79 & 1.838 & 11.57 & 100 \\
\hline LCD-SVD -CRO-SVM3 & 30 & 10 & 817.97 & 290.05 & 0.65 & 100 \\
\hline LCD-SVD-GA-SVM3 & 30 & 10 & 163.75 & 20.2546 & 8.64 & 100 \\
\hline LCD-SVD-PSO-SVM3 & 30 & 10 & 3303.89 & 3.42 & 7.23 & 100 \\
\hline
\end{tabular}

TABLE 4: Identification results of 2nd data based on LCD-SVD and CRO-SVM method.

\begin{tabular}{|c|c|c|c|c|c|c|c|c|}
\hline Test samples & \multicolumn{4}{|c|}{ Fault feature vector } & \multirow{2}{*}{$\begin{array}{c}\text { LCD-SVD-CRO-SVM1 } \\
(+1)\end{array}$} & \multirow{2}{*}{ LCD-SVD-CRO-SVM2 } & \multirow[t]{2}{*}{ LCD-SVD-CRO-SVM3 } & \multirow{2}{*}{$\begin{array}{c}\begin{array}{c}\text { Identification } \\
\text { results }\end{array} \\
\text { IR fault }\end{array}$} \\
\hline IR fault & 3.7993 & 1.3423 & 0.6323 & 0.5431 & & & & \\
\hline IR fault & 4.6628 & 1.5491 & 0.7317 & 0.6030 & $(+1)$ & & & IR fault \\
\hline Ball fault & 6.6009 & 1.2908 & 0.8887 & 0.6388 & $(-1)$ & $(+1)$ & & Ball fault \\
\hline Ball fault & 6.0625 & 1.1805 & 0.8026 & 0.7126 & $(-1)$ & $(+1)$ & & Ball fault \\
\hline Normal & 1.2331 & 0.9392 & 0.4472 & 0.3926 & $(-1)$ & $(-1)$ & $(+1)$ & Normal \\
\hline Normal & 1.1058 & 0.8236 & 0.6051 & 0.4798 & $(-1)$ & $(-1)$ & $(+1)$ & Normal \\
\hline OR fault & 11.2975 & 3.0615 & 2.0807 & 1.3404 & $(-1)$ & $(-1)$ & $(-1)$ & OR fault \\
\hline OR fault & 9.8392 & 2.8869 & 2.0738 & 1.6064 & $(-1)$ & $(-1)$ & $(-1)$ & OR fault \\
\hline
\end{tabular}


fault diagnosis of roller bearing. Furthermore, the proposed method could be applied to other parts of rotating machinery.

\section{Conflict of Interests}

The authors declare that there is no conflict of interests regarding the publication of this paper.

\section{Acknowledgments}

This research is supported by Chinese National Science Foundation Grant (no. 51075131), the grant of the 12th Five-Year Plan for the construct program of the key discipline (Mechanical Design and Theory) in Hunan province (XJF2011[76]), Cooperative Demonstration Base of Universities in Hunan "R \& D and Industrialization of Rock Drilling Machines" (XJT [2014] 239), and scientific research project of Hunan Education Department (no. 14C0789).

\section{References}

[1] R. B. Randall and J. Antoni, "Rolling element bearing diagnostics-a tutorial," Mechanical Systems and Signal Processing, vol. 25, no. 2, pp. 485-520, 2011.

[2] B. Assaad, M. Eltabach, and J. Antoni, "Vibration based condition monitoring of a multistage epicyclic gearbox in lifting cranes," Mechanical Systems and Signal Processing, vol. 42, no. 1-2, pp. 351-367, 2014.

[3] Z. Li, Z. Ma, Y. Liu, W. Teng, and R. Jiang, "Crack fault detection for a gearbox using discrete wavelet transform and an adaptive resonance theory neural network," Journal of Mechanical Engineering, vol. 61, no. 1, pp. 63-73, 2015.

[4] B. Muruganatham, M. A. Sanjith, B. Krishnakumar, and S. A. V. Satya Murty, "Roller element bearing fault diagnosis using singular spectrum analysis," Mechanical Systems and Signal Processing, vol. 35, no. 1-2, pp. 150-166, 2013.

[5] B. Kilundu, X. Chiementin, and P. Dehombreux, "Singular spectrum analysis for bearing defect detection," Journal of Vibration and Acoustics, Transactions of the ASME, vol. 133, no. 5, Article ID 051007, 2011.

[6] J. Cheng, D. Yu, J. Tang, and Y. Yang, "Application of SVM and SVD technique based on EMD to the fault diagnosis of the rotating machinery," Shock and Vibration, vol. 16, no. 1, pp. 8998, 2009.

[7] N. E. Huang, Z. Shen, S. R. Long et al., "The empirical mode decomposition and the Hilbert spectrum for nonlinear and non-stationary time series analysis," Proceedings of the Royal Society A, vol. 454, no. 1971, pp. 903-995, 1998.

[8] J. S. Smith, "The local mean decomposition and its application to EEG perception data," Journal of the Royal Society Interface, vol. 2, no. 5, pp. 443-454, 2005.

[9] G. Rilling and P. Flandrin, "One or two frequencies? The empirical mode decomposition answers," IEEE Transactions on Signal Processing, vol. 56, no. 1, pp. 85-95, 2008.

[10] Z. Wu and N. E. Huang, "A study of the characteristics of white noise using the empirical mode decomposition method," Proceedings of the Royal Society A: Mathematical, Physical and Engineering Sciences, vol. 460, no. 2046, pp. 1597-1611, 2004.
[11] J. Cheng, D. Yu, and Y. Yang, "Application of support vector regression machines to the processing of end effects of HilbertHuang transform," Mechanical Systems and Signal Processing, vol. 21, no. 3, pp. 1197-1211, 2007.

[12] J.-S. Cheng, J.-D. Zheng, and Y. Yang, "A nonstationary signal analysis approach-the local characteristic-scale decomposition method," Journal of Vibration Engineering, vol. 25, no. 2, pp. 215-220, 2012.

[13] J. Zheng, J. Cheng, and Y. Yang, "A rolling bearing fault diagnosis approach based on LCD and fuzzy entropy," Mechanism and Machine Theory, vol. 70, pp. 441-453, 2013.

[14] Y. Lei and M. J. Zuo, "Gear crack level identification based on weighted $\mathrm{K}$ nearest neighbor classification algorithm," Mechanical Systems and Signal Processing, vol. 23, no. 5, pp. 1535-1547, 2009.

[15] C.-C. Wang, Y. Kang, P.-C. Shen, Y.-P. Chang, and Y.-L. Chung, "Applications of fault diagnosis in rotating machinery by using time series analysis with neural network," Expert Systems with Applications, vol. 37, no. 2, pp. 1696-1702, 2010.

[16] X. Zhang and J. Zhou, "Multi-fault diagnosis for rolling element bearings based on ensemble empirical mode decomposition and optimized support vector machines," Mechanical Systems and Signal Processing, vol. 41, no. 1-2, pp. 127-140, 2013.

[17] S.-W. Fei and X.-B. Zhang, "Fault diagnosis of power transformer based on support vector machine with genetic algorithm," Expert Systems with Applications, vol. 36, no. 8, pp. 11352-11357, 2009.

[18] H. Xu and G. Chen, "An intelligent fault identification method of rolling bearings based on LSSVM optimized by improved PSO," Mechanical Systems and Signal Processing, vol. 35, no. 12, pp. 167-175, 2013.

[19] V. N. Vapnik, Statistical Learning Theory, John Wiley \& Sons, 1998.

[20] S. Abe, Advances in Pattern Recognition, Springer, London, UK, 2005.

[21] F. Friedrichs and C. Igel, "Evolutionary tuning of multiple SVM parameters," Neurocomputing, vol. 64, no. 1-4, pp. 107-117, 2005.

[22] X. L. Zhang, X. F. Chen, and Z. J. He, "An ACO-based algorithm for parameter optimization of support vector machines," Expert Systems with Applications, vol. 37, no. 9, pp. 6618-6628, 2010.

[23] A. Y. S. Lam and V. O. K. Li, "Chemical-reaction-inspired metaheuristic for optimization," IEEE Transactions on Evolutionary Computation, vol. 14, no. 3, pp. 381-399, 2010.

[24] A. Y. S. Lam and V. O. K. Li, "Chemical reaction optimization: a tutorial," Memetic Computing, vol. 4, no. 1, pp. 3-17, 2012.

[25] Z. Shen, X. Chen, X. Zhang, and Z. He, "A novel intelligent gear fault diagnosis model based on EMD and multi-class TSVM," Measurement, vol. 45, no. 1, pp. 30-40, 2012.

[26] H. L. Ao, J. Cheng, K. Li, and T. K. Truong, "A roller bearing fault diagnosis method based on LCD energy entropy and ACROASVM," Shock and Vibration, vol. 2014, Article ID 825825, 12 pages, 2014.

[27] H. Ao, J. Cheng, J. Zheng, and T. K. Truong, "Roller bearing fault diagnosis method based on chemical reaction optimization and support vector machine," Journal of Computing in Civil Engineering, Article ID 04014077, 2014. 

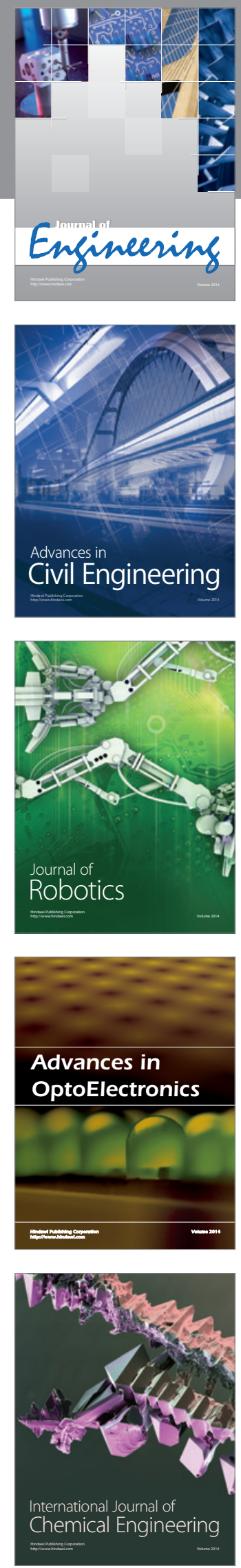

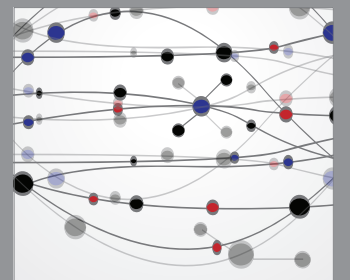

The Scientific World Journal
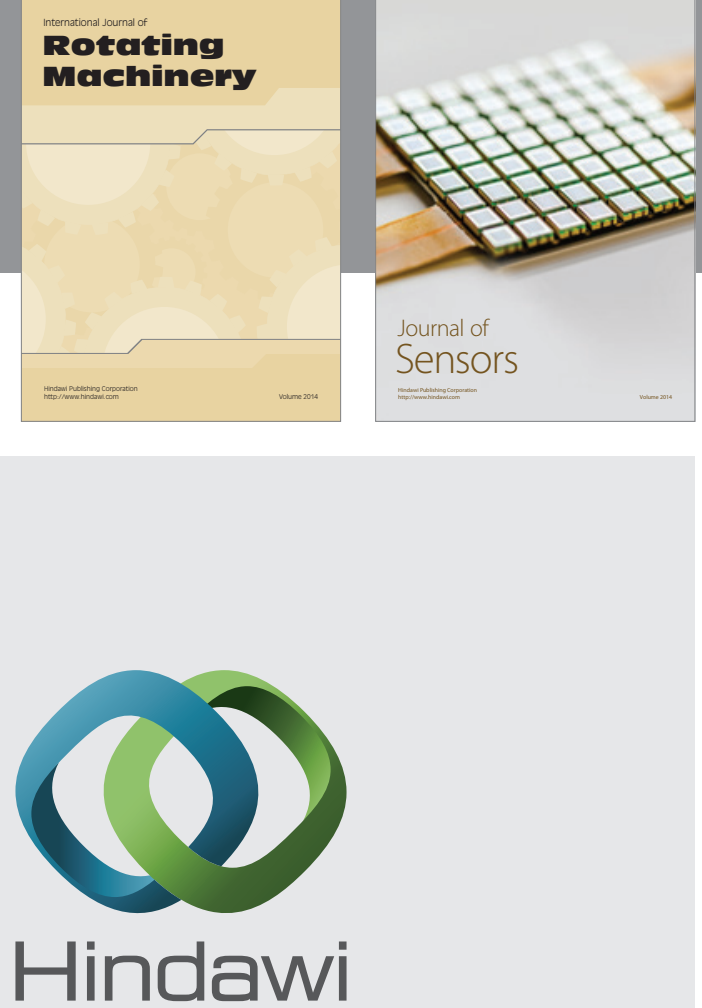

Submit your manuscripts at http://www.hindawi.com
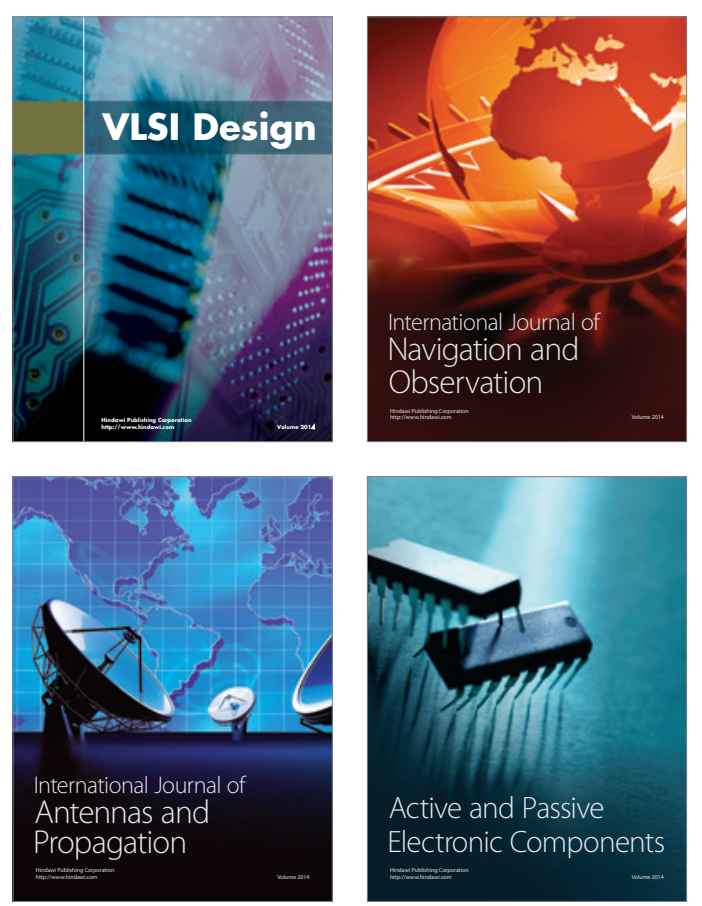
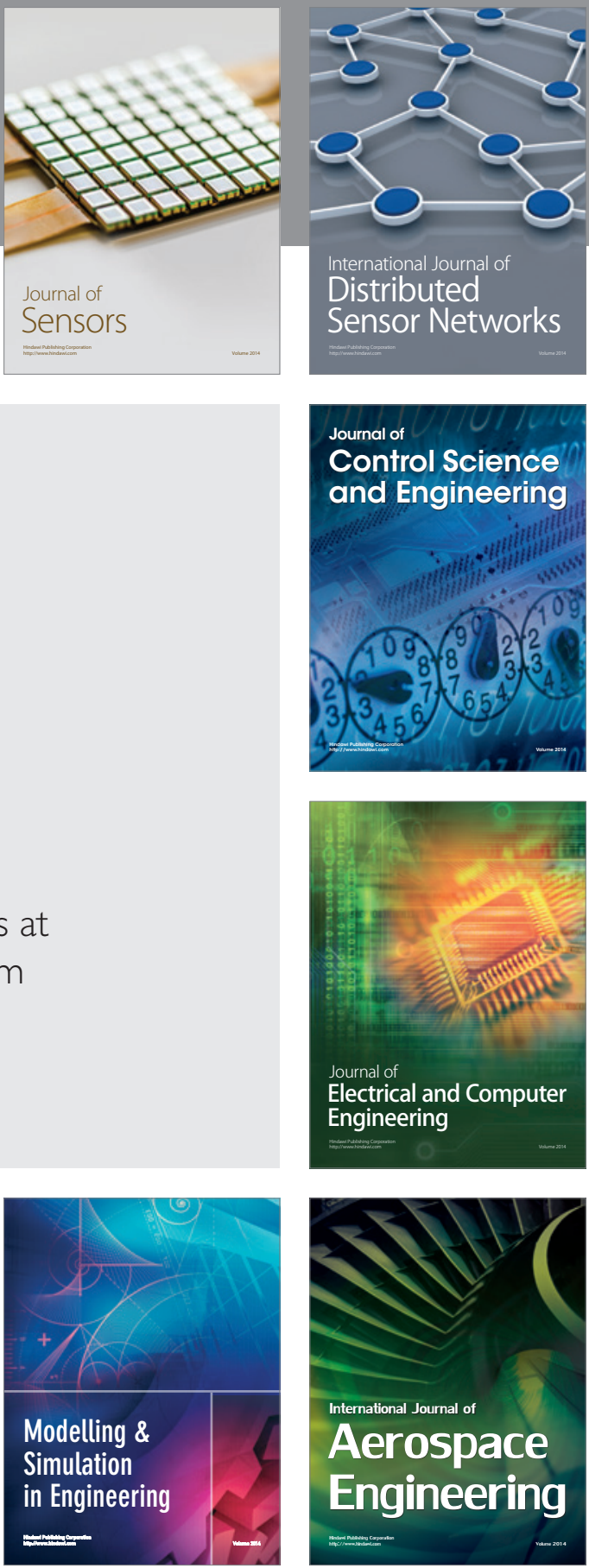

Journal of

Control Science

and Engineering
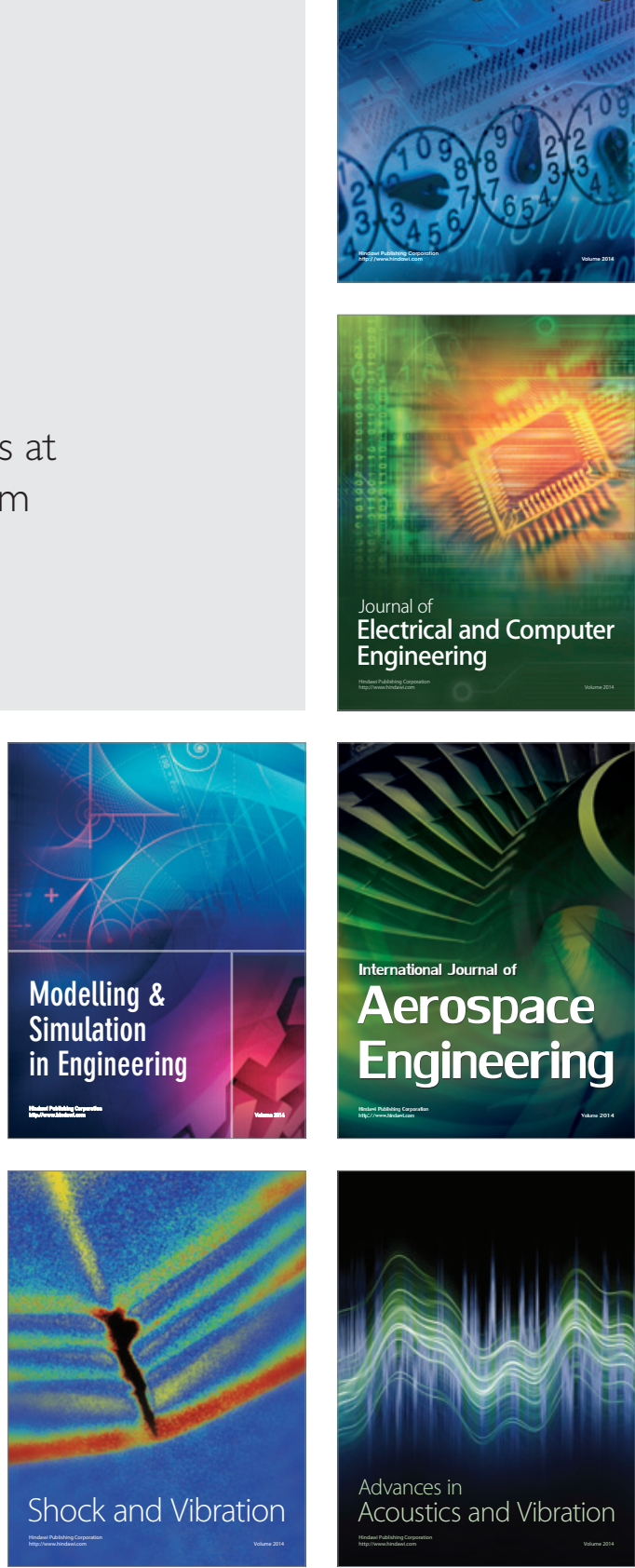